\title{
Research and Design of Virtual Oscilloscope Based on Android_QT Hong Zou ${ }^{1, a}$, Xiangjiao shen ${ }^{1, b}$ and Guoping Chen ${ }^{1, c}$ \\ ${ }^{1}$ School of Chongqing University of Posts and Telecommunications, Chongqing 400065, China; \\ a zouhong@cqupt.edu.cn, ${ }^{b} 13032361163 @ 163 . c o m,{ }^{c}$ chengp@cqupt.edu.cn
}

Keywords: Android, QT, Virtual Oscilloscope, Signal Acquisition.

\begin{abstract}
First of all, the paper would be introduce the study on portable virtual instrument technology based on the Android platform, then particularly in virtual oscilloscope as an example, focusing on the overall design of the system. Stratified in different signal acquisition system in order to meet the demands for different users; Using QT for efficient and flexible design of soft Panel, function modules and filters. The design can input dual-channel analog signal, then achieve the basic functions of an oscilloscope, like, set certain control parameters, real-time data acquisition, processing, display and storage.
\end{abstract}

\section{Introduction}

Dating back to the early 20th century, electronic measuring instruments can be divided into four stages: analog instruments, digital instruments, intelligent instruments and virtual instruments[1]. Basic structure of the analog instruments are electromagnetic mechanical simulations, which using the pointer shows the final result. The core idea of digital instruments is converting analog signals to digital signals, and outputting final results in digital form. The core components of intelligent instruments is MCU, who use single-chip microcomputer to realize co-ordination and control of the instrument functions. Although the technology and performance of these instruments have been mature, but it has some shortcomings, such as, larger size caused difficulties to carry, fixed functions, and expensive[2]. With the development of the times and computer technology, NI company[3] proposed the concept of virtual instruments, which is a huge breakthrough to traditional concept of experimental equipment. It proposes embedded operating system into instruments, in that way, implementing the flexible control of measuring instruments by operating systems application software .

Virtual instruments consists of three main components, high-performance hardware modular, efficient and flexible software and hardware and software platform for integrated, to complete a variety of test, measurement and automation applications. In a virtual system, many modules of the system are implemented by software, so users can easily according to their own needs to implement corresponding features by using different algorithms[4]. Compared with traditional instruments, the technology enhances system flexibility and processing efficiency, save development time and material resources, and realization of human-computer interaction and interface design.

\section{Design of virtual oscilloscope}

Today, most of instruments realized with LabVIEW, which belongs to NI company. Taking graphical language and easy writing advantages of LabVIEW to design PC virtual instrument interface, combined with data acquisition card and sensors for data acquisition and processing[5][6].

The design of virtual oscilloscope consists of hardware and software design. The part of hardware uses STM32F4 series micro-controller as the main control chip, with both the internal and external A/D to sample the analog signal, master-slave structure of USB for system data transferring, and a SD card for data storage. Software includes drivers and software Panel, designing efficient and flexible soft Panel, function modules and filters with QT. 
Hardware design of oscilloscope. According to the functional requirements of the system, hardware design including analog data acquisition, A/D conversion, data storage, data transfer, the system shown in Fig. 1.

In the design, the main control flow of STM32F4, Android soft Panel issues control command to STM32F4 to set different frequencies of sampling rate, then STM32F4 adjusts the sampling rate of the $\mathrm{AD}$, while change the DMA packet size. Packaging converted data to buffer through DMA way, , then using USB-OTG line transmitting data to mobile phone when the buffer is full. In the way, adapting to the different data processing abilities which comes from different properties phone to the USB port, And finally, displaying the signal on Android software Panel.

Oscilloscope software design. The design will take full advantage of QT software development platform, using QT threads design of signal collection, filter design, synchronous processing parameters measurement and waveform display.

After understanding the functionality and performance of the oscilloscope, determining the design of the virtual oscilloscope module structure and function according to the design of the virtual oscilloscope's design requirements. System software flow chart shown in Fig. 2.

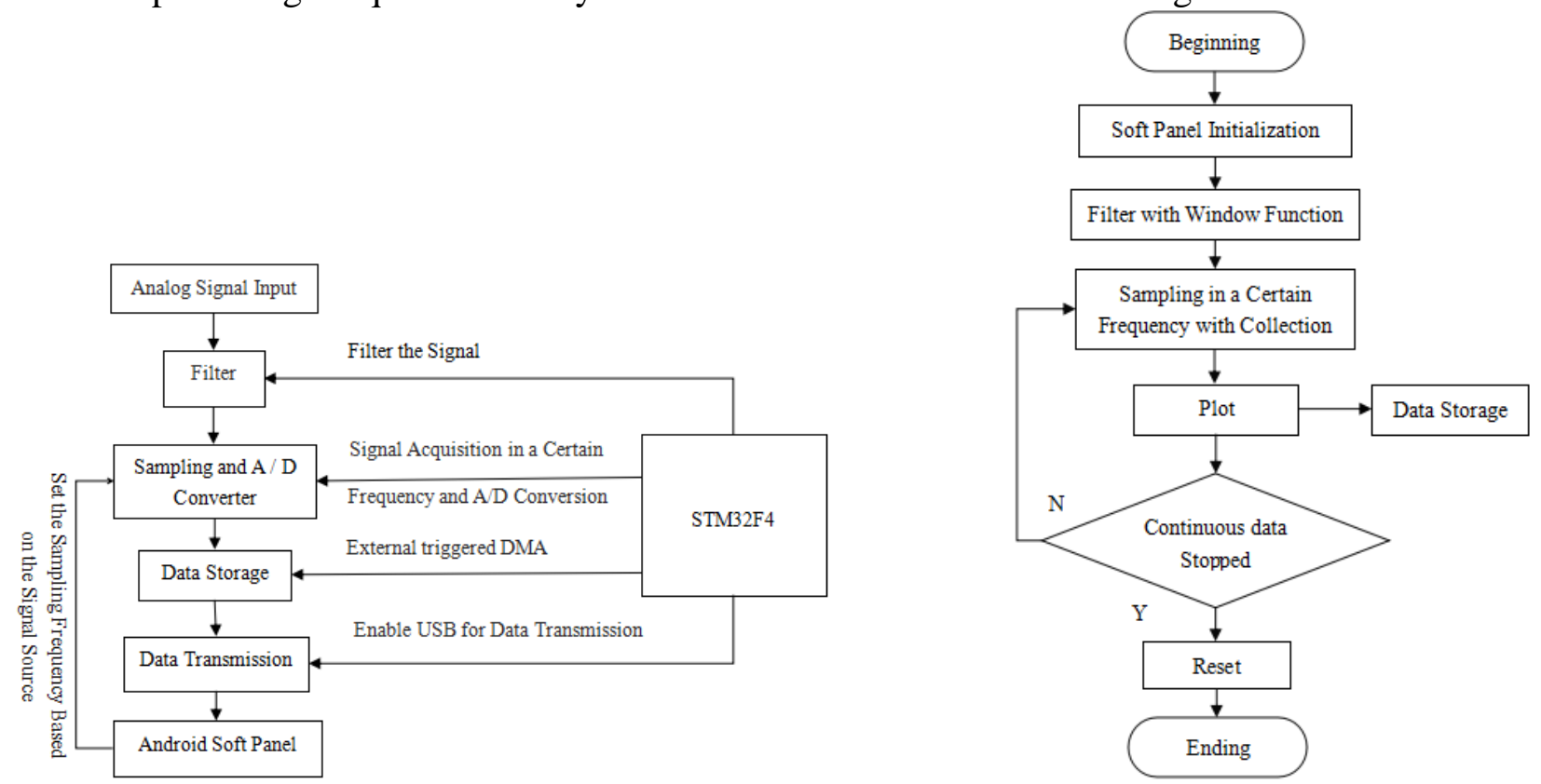

Fig. 1 System Architecture Diagram

Fig. 2 System Software Design Flow Chat

The design and realization for each functional module. Qt interacts the outside world with signals and slots mechanism . Signals and slots is a safe and reliable way, it is equivalent to a function pointer, supporting for collaborative work between objects without knowing each other.

In the paper, the design and realization for each module is shown below

(1) Interface Control Module. Triggered control including trigger source, rising edge triggered, and falling edge trigger, for real-time capture of signal waveform which meets triggered conditions; Channel selection control includes channel A and channel B, it is used to select the input signal channel; Parameters measurement including site value, and frequency, respectively measuring all channel signal of site value and frequency of the maximum, minimum, and mean values;

Interface control module, including initializing, the implementation of slot functions related to the change for the signal amplitude and frequency parameters, defining a pointer to draw waveform.

class MainWindow : public QWidget

\{

Q_OBJECT

public:

MainWindow( QWidget * = NULL );

void start();

double amplitude() const; 
double frequency() const;

private:

Plot *d_plot; //pointer to draw waveform

QByteArray m_buffer; //stored audio data

private slots:

void AnalogProperty(); //analog Property setting

void CursorsProperty(); //cursors Property setting

void MeasureProperty(); //measure Property setting

\} ;

void TriggerProperty(); //trigger Property setting

(2) Data Sampling Module. The channels for A/D converter in ADC can be single, sequential, scan or a break mode. In the paper, it would be set as single-conversion mode. Creating a sample thread and then setting sample interval to realization data sampling.

class SamplingThread: public QwtSamplingThread

\{

Q_OBJECT

public:

SamplingThread( QObject * parent = NULL );

protected:

virtual void sample( double elapsed ); //set sample interval

public Q_SLOTS:

void value( qint64, const QByteArray \&);//receive data should be draw on the Panel $\$ private: ;

QByteArray m_buffer; //receive data

(3) Waveform drawing. QWT is used to draw waveform. QWT is form based on 2D components to display data, the data source can be numerical, array, or a set of floating point numbers, and so on. In the paper, the $\mathrm{AD}$ sampling data will be real-time saved in buffer. Using the QwtPlotCanvas class to draw a QwtPlot view window, and then read data from the buffer to draw waveform.

class Plot: public QwtPlot

\{

Q_OBJECT

public:

void start(); //start to plot the waveform

virtual void replot(); //replot the waveplot

public Q_SLOTS:

void setIntervalLength( double ); //update axis

private:

void updateCurve(); //update curve

void incrementInterval(); //scale conversation

private:

QwtPlotMarker *d_origin; //a point to draw marker

;

QwtPlotCurve *d_curve; //a point to draw curve

(4) Waveform storage. Data storage methods in QT can be a file or a database. In the paper, it will capture screenshots with the function of QPixmap::grabWindow in the Qt for waveform storage.

\section{Analysis of test results}

After the completion of each functional module design of virtual oscilloscope, the oscilloscope software Panel should be tested. Importing two-channel signal, square wave and sine wave, whose 
peak respectively is $\mathrm{VA}=100 \mathrm{mV}, \mathrm{VB}=100 \mathrm{mV}$. Accessing to the phone, and finally displayed as shown in the following figure.

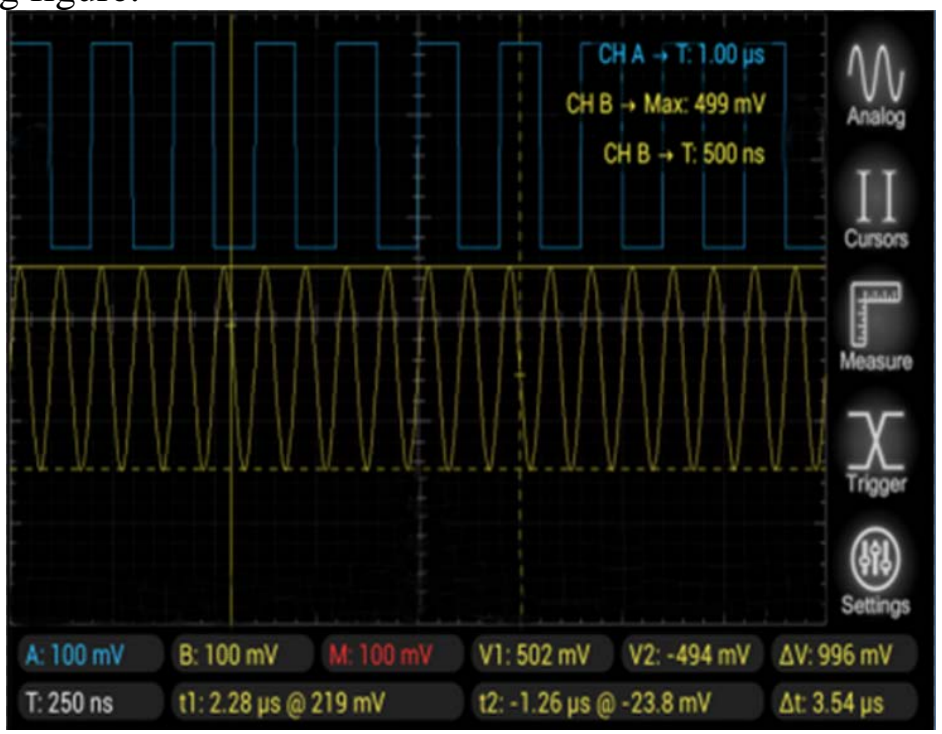

Fig. 3 Screenshots on mobile phone

\section{Summary}

Present situation of the development of the Android operating system, combining with the thought of designing virtual instrument based on LibView, the paper proposes a way to research and design of portable virtual instrument based on Android, using the highest frequency used oscilloscope as an example. For the implementation of the design, whether for industrial or school teaching all has significance. Traditional instruments such as Oscilloscopes, signal sources are too heavy, cannot be moved to all classrooms, especially those are very expensive, thus the experimental equipment are often equipped not fully enough, causing the separation of theory and experiment course. For Android devices, each student is not familiar with, therefore, designing an Android-based portable measuring instruments can be used the situation that lacks of laboratory equipment, in this way the problem of separation of students' comprehensive ability and practical skills can be solved very well.

In the paper, it only implement the basic functions of oscilloscope. As we all know, there are many kinds of measuring instruments, therefore, by increasing the realization, like, sweeper, signal generator and a series of measuring instruments to complete this application, in order to meet the different needs of different users.

\section{References}

[1] Wu Xinghua, Wang xuming. Status and Prospect of Research on Application of Virtual Instrument Technology in China [D]. Modern Scientific Instrument. 2011.8

[2] Wang Shuo, Wang Bing, Zou Guangnan. Design and Implementation of Virtual Instrument Based on LabVIEW and Sound Card[J]. Electronic Design Engineering. 2014.5

[3] Han Shuo, Zhao Lianjuan. Design of Multi-function Oscilloscope Based on Virtual Instrument Technology [J]. The Science and technology wind. 2014.3

[4] Lu Yafeng, Chen Yijun. Research Status and Prospects of Virtual Instruments [D]. Theory and Methods. 2010.11

[5] Xiao Yuan, MA Li-ping. Design and Implementation of Virtual Oscilloscope Based on LabVIEW and Sound Card[J]. Journal of Shanxi University of Science \& Technology(Natural Science Edition:2009, 02, pp. 023. 
[6] Yang Fengping, Jiang Zhiling. Design of Oscilloscope Based on Virtual Instrument Technology[J]. Journal of East China Jiaotong University, 2009, 01, pp. 012. 